\title{
INHABIT - information and service microarchitecture for University campus
}

XXIV International Conference of the Iberoamerican Society of Digital Graphics Medellin | Colombia

\author{
Silvia Patricia Hernández \\ FAUD, UNC | Argentina | patricia.hernandez@unc.edu.ar \\ Cristina Chaves \\ FAUD, UNC | Argentina | cristinacha@hotmail.com \\ Luciana Lanzone \\ FAUD, UNC | Argentina | luciana.lanzone@unc.edu.ar \\ Lucía Ron \\ FAUD, UNC | Argentina | lucia.ron@unc.edu.ar
}

\begin{abstract}
This research team rethought a microarchitecture design with service for the entire community of the city of Córdoba at various sites in it. A real space is proposed that territorializes the digital, designed and built with new technologies with the aim of spreading to the city all the open activities carried out by the area of culture and extension of the National University of Córdoba, through the incorporation of technological means that are identified with the application of home automation and sustainability. The plan includes a network of microarchitectures throughout the city, beginning with the location on campus.
\end{abstract}

Keywords: Microarchitecture; Inclusivity; New technologies; Comfort.

\section{INTRODUCTION}

Continuando la línea de investigación orientada al diseño y desarrollo de microarquitecturas inmóticas, sustentables e inclusivas que ha trabajado este equipo por varios años, se presentan los avances obtenidos en la etapa más reciente de trabajo. Buscando desarrollar dispositivos arquitectónicos concretos, que transformen los espacios públicos en los que se instalan y activen dinámicas participativas e inclusivas con sus usuarios, el proyecto presentado trabaja un diseño de microarquitecturas que modifican la sinergia entre dos "ciudades", la ciudad universitaria y estudiantil dentro de la ciudad de Córdoba.

Partiendo de los resultados obtenidos en etapas anteriores, en esta ocasión se complejizó el programa funcional para que responda a necesidades detectadas a escala local y urbana, integrando los conceptos de inclusión, articulación y organización. Siendo el lugar de trabajo de este equipo la ciudad de Córdoba (Argentina), se determinó esa ciudad como lugar de implantación del proyecto, a la vez que su situación sociocultural concreta se adoptó como disparadora del programa funcional. Un estudio previo sobre las distintas actividades culturales que convocan diversas instituciones públicas en la ciudad determinó como programa estratégico a abordar la difusión en toda la ciudad de las actividades abiertas (y muchas veces gratuitas) realizadas por el área de cultura de la Subsecretaría de Extensión de la Universidad Nacional de Córdoba (Figura 1).
La Universidad Nacional de Córdoba es la segunda universidad más antigua de Latinoamérica. Cuenta con 125.878 alumnos, que junto a docentes y no-docentes superan las 180.000 personas. Esta población universitaria es de suma importancia relativa en una ciudad como Córdoba, que cuenta con 1.700 .00 habitantes proyectados al 2020. Debido a esto, las actividades y programas que realiza la Subsecretaría de Cultura de Extensión contempla acciones muy heterogéneas de artistas profesionales y vocacionales, tanto locales, nacionales como internacionales, ya sean integrantes de la comunidad de la universidad o de diversas procedencias y espacios. A lo largo del año se realizan numerosos cursos, capacitaciones, exposiciones, teatro, danzas, ferias, recitales, etc. Sin embargo, se detectó la necesidad de ampliar la convocatoria y difusión de muchos eventos que suele estar restringida por los fondos específicos con que cuenta esa institución. Se planteó entonces un diseño de microarquitectura en red, que sirva como difusores para toda la comunidad de la ciudad de Córdoba. La red está formada por varios dispositivos ubicados en el territorio, con un diseño y funciones determinadas con base en las distintas estrategias diseñadas y los contextos de localización individual. De esta forma, la red (imagen 6) amplía el alcance de los mecanismos tradicionales de difusión, con énfasis en las nuevas tecnologías digitales que estructuran una red en el territorio. 


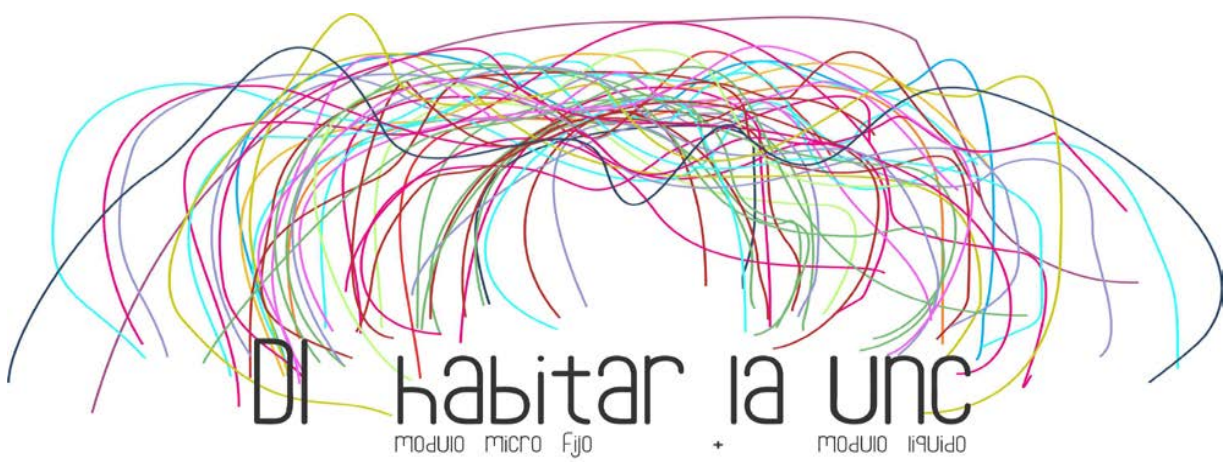

Imagen1: Logo de difusión del proyecto (elaboración propia).

\section{OBJETIVO PARTICULAR}

Concretamente, en este trabajo buscamos diseñar microarquitecturas con tecnologías innovadoras, ubicadas en espacios estratégicos de la ciudad de Córdoba, con la función de completar vías y territorios vacantes en la comunicación de los eventos culturales organizados por la Universidad a través de la incorporación de recursos arquitectónicos/TIC se identifiquen con la aplicación de la inmótica, la sustentabilidad e inclusividad.

\section{SITIO DE IMPLANTACIÓN}

En primer lugar, se elige como locación el campus de la Universidad Nacional de Córdoba (Ciudad Universitaria), ubicado al Sur del área central de la ciudad. Dentro del campus se detectó un espacio en el mismo ingreso desde distintos puntos de la ciudad, conjunción de vías vehiculares (transporte particular y transporte público) y peatonales, y con acceso directo desde el tradicional barrio Nueva Córdoba, consolidado como lugar de residencia de un gran número de estudiantes de la universidad. Esto lo convierte en un paso obligado para la mayoría de los estudiantes, por lo que es denominado Portal de la Universidad. (imagen2) (imagen 3).

El Barrio Nueva Córdoba es un barrio ubicado al sur del área central de Córdoba. Es una de las zonas más pobladas de la ciudad ya que cuenta con más de 40.800 habitantes, de los cuales la mayoría son alumnos universitarios (proyecciones del Instituto Nacional de Estadísticas y Censos al año 2020). La magnitud de su población, así como la gran variedad de comercios y servicios a escala urbana y regional presentes en el barrio lo convierten casi en una ciudad dentro de la otra ciudad. Lo mismo ocurre con el campus de la universidad, llamado ciudad universitaria. El punto de encuentro entre estas dos ciudades es, justamente, el Portal de la Universidad, donde se localiza nuestra propuesta. (imagen4)

\section{PROPUESTA}

La premisa principal que estructura el proceso de diseño es el desarrollo del concepto de interculturalidad como la conexión o intercambio de manera horizontal de diferentes grupos humanos en un espacio de construcción teórico, práctico, reflexivo y de conocimiento, no solo a nivel de

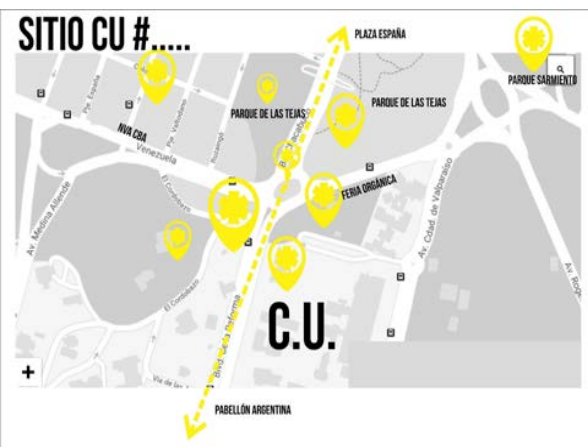

Imagen 2: sitio elegido, Portal de la UNC. (imagen propia)

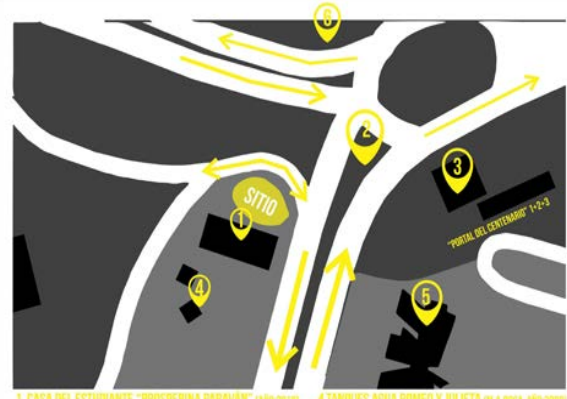

Imagen 3: sitio entorno inmediato, (imagen propia)

capacitación sino también de actividades recreativas y de intercambio cultural.

Para aumentar la difusión y reconocimiento de estas actividades es que hacemos uso de una segunda premisa, que es la incorporación en el espacio físico de los medios tecnológicos que se identifiquen en la aplicación de la domótica, en este caso Inmótica, (CEDOM, 2007) bajo lineamientos de sustentabilidad ambiental y tecnológica, 
pero también de inclusividad, entendida como la que atiende las necesidades de jóvenes, niños, adultos mayores como a personas con capacidades diferentes.

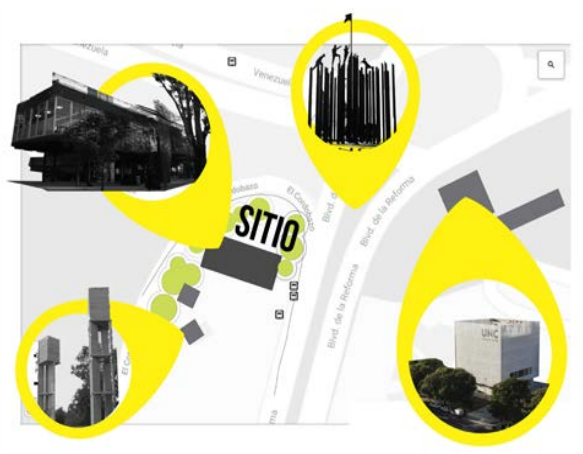

Imagen 4: sitio elegido, entorno inmediato en 3D (imagen propia) Por último, buscamos materializar estos dispositivos en espacios vacíos o anodinos en el sistema espacial del campus universitario, dotándolos de sentido e identidad.

El esquema funcional concreto desarrolla un punto de encuentro entre la ciudad de Córdoba, Nueva Córdoba y la Ciudad Universitaria, que transmita los datos de tópicos, eventos y cursos hacia la ciudad. El dispositivo de microarquitectura difunde acciones que están trasmitiéndose en redes virtuales, cursos de capacitación, eventos y espacios culturales de la UNC, información universitaria, y también diferentes posibilidades e inquietudes que se desarrollen en la ciudad. (imagen 5)

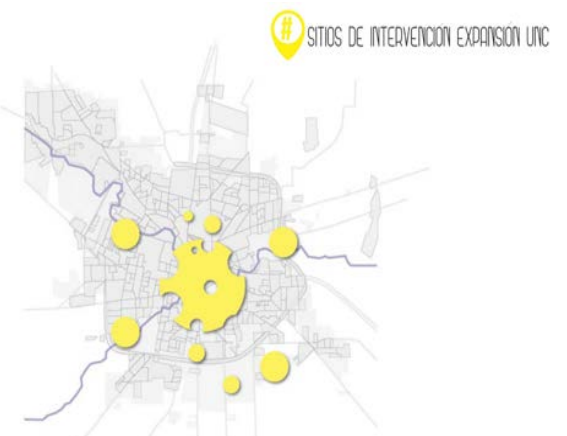

Imagen 5: red de extensión cultural de la UNC en la ciudad

\section{ENFOQUE URBANO Y SOCIAL}

Al decir de Virginia Romanutti, coordinadora de la RED Ciudadana Nuestra Córdoba (2020), las transformaciones necesarias para lograr una ciudad Justa, Democrática y Sustentable requieren de la creación de las condiciones para que las mismas ocurran y requieren también de la responsabilidad colectiva asumida desde distintos sectores. Con este objetivo de trascender los ámbitos de Ciudad Universitaria y llegar a todos los ciudadanos es que interpretamos el accionar colectivo.

Por otro lado, creemos que trabajar con lógicas de trabajo en conjunto o con inteligencia grupal produce resultados más confiables que procesos individuales; de acuerdo a Gutiérrez-Rubí, A, (2010). El trabajo que se maneja en grupo, siempre recibe una mejor aceptación, ya que la sabiduría colectiva da forma y ayuda a formación de empresas y sociedades más sólidas.

Cuando nos referimos a la creación de manera colectiva, en este proyecto es cuando el egresado, o miembro de la comunidad de la universidad participa de los eventos que son para toda la comunidad como cuando un grupo de la comunidad ciudadana actúa en la universidad. Se propone entonces un dispositivo que consolide un espacio de comunidades virtuales formadas, que tendrán su espacio físico en la microarquitectura Habitar para que con la fuerza de comunicación colectiva a través de las redes que ayudan a la sociedad a resolver problemas, a crear espacios colectivos de trabajo, a informarse de las actividades y a interactuar con las mismas, y se incorporen los ciudadanos a las actividades organizadas o propuestas por la universidad. (imagen 6)

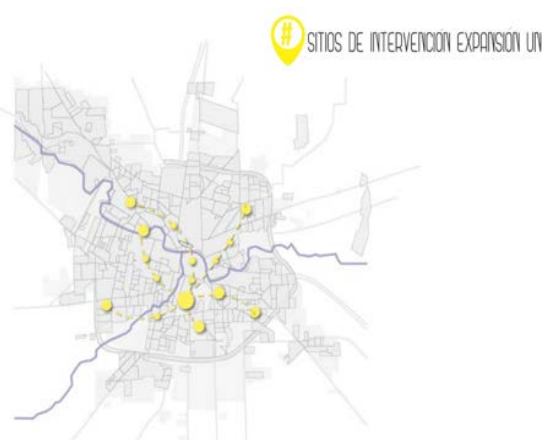

Imagen 6: redes de difusión en la ciudad (imagen propia)

\section{METODOLOGÍA}

Se trabajó con el diseño centrado en el usuario para articular estas microarquitecturas con la ciudad y que se constituyan en un elemento de aporte a los sistemas inteligentes de las ciudades, coincidiendo con la postura de De Los Santos Aransay, A., (2009). Pretendemos que estas micro permitan caracterizar en forma óptima las necesidades de los usuarios. Pretendemos también responder con el diseño del equipo y de las envolventes a los requerimientos, no sólo a nivel formal sino que se constituyan en un aporte posibilitante a la interacción planteada, Usuario/Espacio. Esta metodología de diseño centrado en el usuario se inserta en las metodologías de diseño que contemplan, tanto la Planificación, como el Diseño del Objeto Arquitectónico Inclusivo, y la Construcción (con tecnología local).

Para lo cual se requiere de las sub-etapas de Análisis, Determinación de requerimientos, Ensayos de propuestas, Desarrollo de proyecto y luego Verificación con asesores. Proponemos en esta etapa el trabajo con modelos virtuales.

La premisa de creación colectiva condujo a iniciar cada etapa del proceso con encuestas on-line distribuidas por el método bola de nieve, donde se consultaba sobre sitios posibles de implantación y sobre las premisas funcionales del dispositivo. El proceso de diseño busca una 
arquitectura líquida pero física, transformable y adaptable, mutante de acuerdo a los requerimientos

Esto conlleva otra etapa metodológica muy importante que es la definición del proyecto con la tecnología. Esta etapa está en desarrollo. La construcción física de la obra se produce a partir de cualquier material, medio y procedimiento. Este equipo busca materiales maleables que nos permitan la ductilidad y mutación, que conlleven tecnologías como colectores solares, como las cubiertas de Morales Guzmán, C. (2014). Estos objetivos nos marcan un camino de exploración de las posibilidades en los materiales y su transformación. Una vez que se agotan las posibilidades del mercado se inicia un recíclaje de formas, objetos y materiales. Es decir, intentaremos con nuevos materiales y la adaptación de los existentes, entre ellos la Nanocelulosa, el Grafeno, las telas tensadas o los Polímeros avanzados o técnicos. Esta etapa la estamos transitando.

Este trabajo, entonces, se enfoca en una búsqueda de materiales innovadores que permitan la mutación, y que a la vez permitan la incorporación de la tecnología. También se priorizó la sustentabilidad técnica y tecnológica, de forma que los dispositivos pudieran ser construidos con recursos y mano de obra locales, aprovechando incluso el desarrollo de I+D en inmótica en la misma universidad.

La intención es no sólo ofrecer un producto a la comunidad universitaria, sino también al gobierno de la ciudad. Se realizó el legajo técnico con diseño paramétrico, perspectivas axonométricas y maquetas digitales para lograr una aproximación a la realidad y que puedan ser construidos.

\section{DISEÑO DEL DISPOSITIVO}

La microarquitectura que se diseñó es experiencial, flexible, pensando en la Arquitectura Líquida que se está desarrollando en un continuum. El término Arquitectura Líquida fue acuñado por el arquitecto, diseñador y artista Marcos Novak en la década de los 90. Novak (2004) afirma que la arquitectura líquida es una especie radical de sinfonía del espacio, cuya característica principal es que jamás deja de desarrollarse, hasta transformarse en su propio refugio. Otro concepto que se trabajó es también planteado por Novak, cuando define la Trans-arquitectura, como la intersección entre información y mundo material, y que se manifiesta como la disciplina que trata de espacios que se transforman a sí mismos.

Novak es sin duda controvertido, y a pesar de esto vanguardistas como Zaha Hadid o Frank Gehry toman luego sus conceptos. Tan cuestionado Novak, sin embargo Bauman (2000 y 2007) desafía esa posibilidad precisando estas etapas de la modernidad para explicar dos momentos de su historia que tienen que ver con la solidez y la liquidez con que la sociedad define sus patrones de conducta. (Imagen 2)

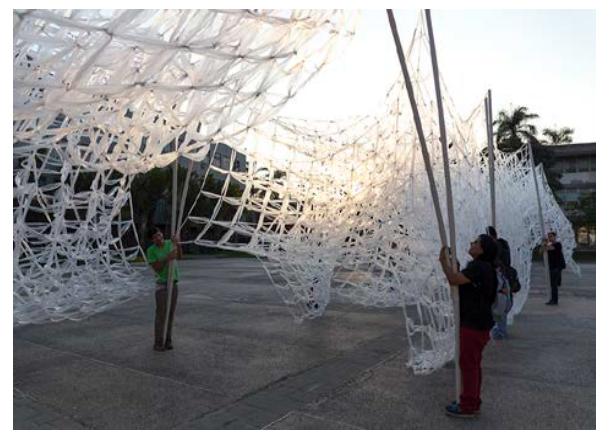

Imagen7: Miguel Braceli, Arquitectura líquida. (plataforma arquitectura)

La arquitectura líquida se puede pretender como la posibilitante de que la forma sea modificada según las necesidades del usuario (facilitando la interacción usuario/espacio/equipos) o respondiendo a un programa de diseño que integre la mutación. (imagen 8)

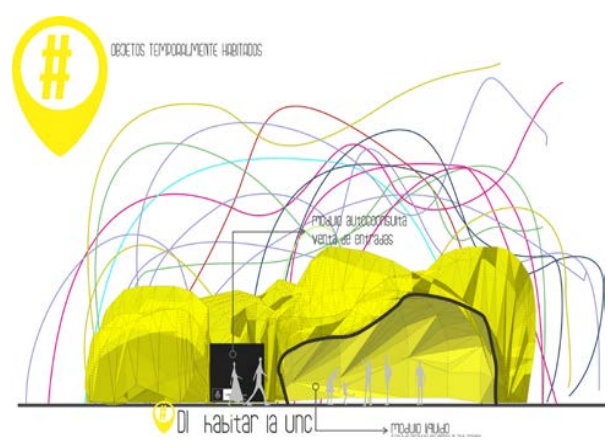

Imagen 8: propuesta física de microarquitectura mutante (imagen propia)

Nuestra propuesta se acerca a estas proyecciones, como también al habitar híbrido, donde Requena (2019) contempla también una arquitectura transformable, real y física, con información interactiva para los usuarios. Lo que evidencia es que apelamos a cambiar y beneficiar a la sociedad con los medios que ya existen, proponiendo la conjunción de lo digital y de la cultura de la universidad abierta a la sociedad entera, en un espacio físico.

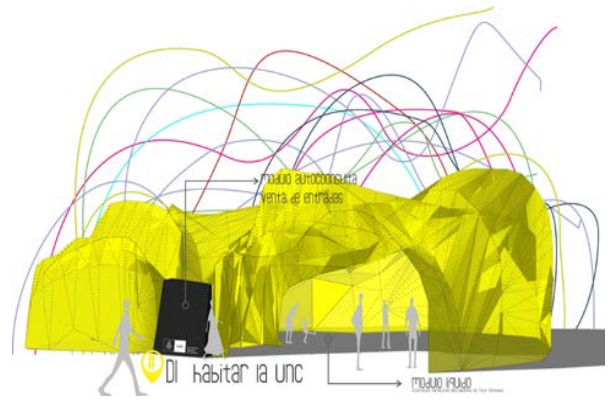

Imagen 9: propuesta física visualización del puesto de venta de entradas y autoconsulta (imagen propia). 


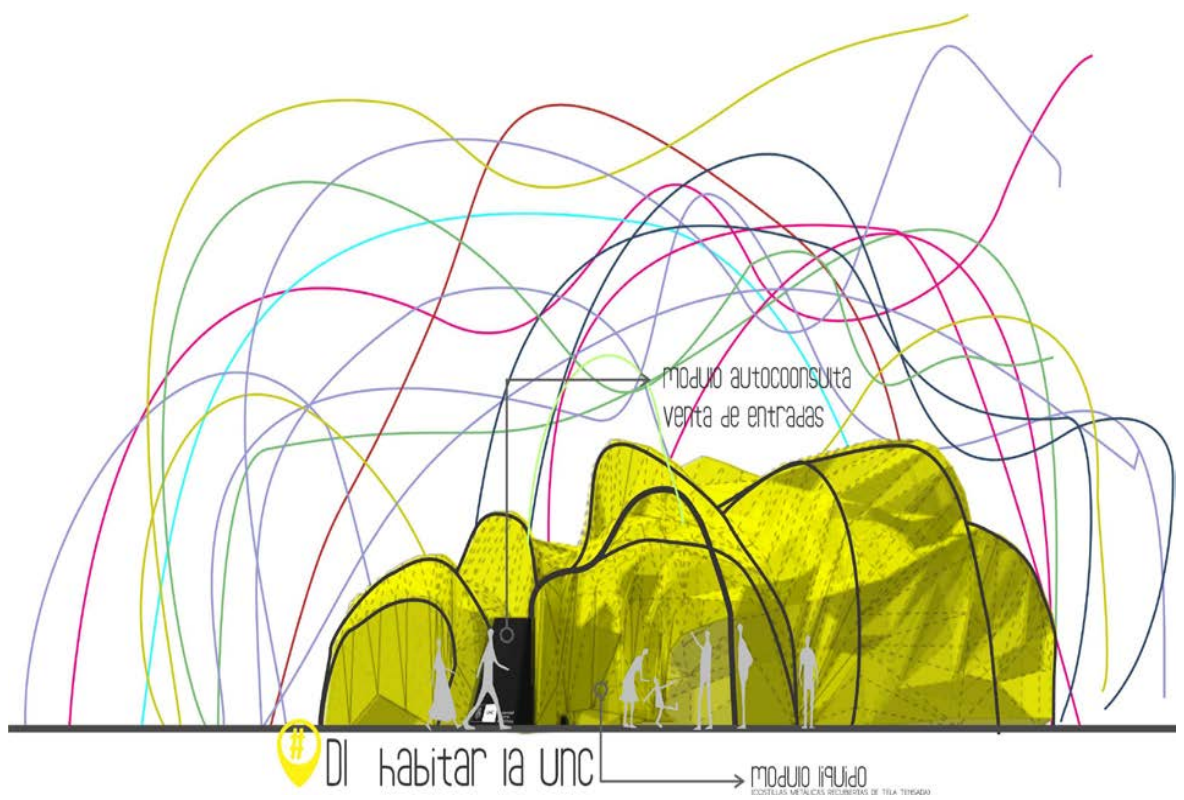

Imagen 10: propuesta física cerrada, antivandalismo (imagen propia)

Entendemos que con sólo introducir una modificación, por pequeña que sea, en algún sector de un sistema, lo que cambia y asimila esa modificación es el sistema entero.

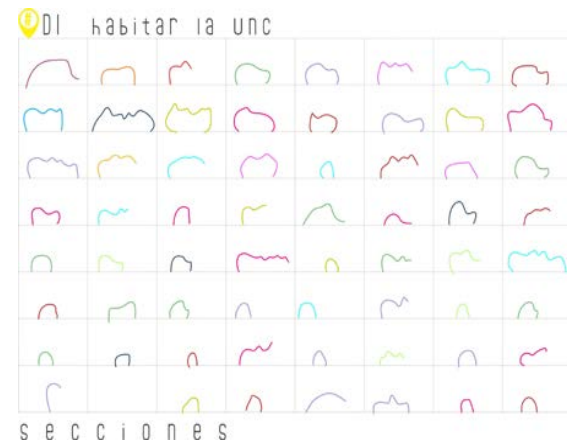

Imagen 11: propuesta perfiles posibles de la micro mutante (imagen propia)

La inclusividad desnuda problemas que fueron manifestados en las encuestas a los usuarios: muchos de ellos no pueden fácilmente encontrar la información de cultura de la universidad. Por lo tanto este sitio les brindará información digital determinada en situaciones espaciales emergentes, con pantallas donde se pueda consultar e interactuar, para ver e imprimir información de cada actividad que se realice, proyecciones $2 \mathrm{D}$ y $3 \mathrm{D}$ sobre las materiales de cobertura, con espacios planteados y pensados en la inclusión social y de diferentes capacidades. (imagen11)

Se proponen también equipos de servicio para imprimir tickets para los shows o exposiciones parar los sean gratuitos, (por lugares y organización) y para abonar los Eventos que son pagos (imagen 12, 13 Y 14).

El material fundamental e innovador son cubiertas (tipo telas) resistentes para el exterior y que permiten incorporar las pantallas oled. (Morales Guzmán, C. (2014). Trabajamos con estas cubiertas como las del instituto de Georgia, (EEUU) que han diseñado una nueva tela que puede generar electricidad a partir de la luz solar y del movimiento. Estos investigadores han dado un paso más

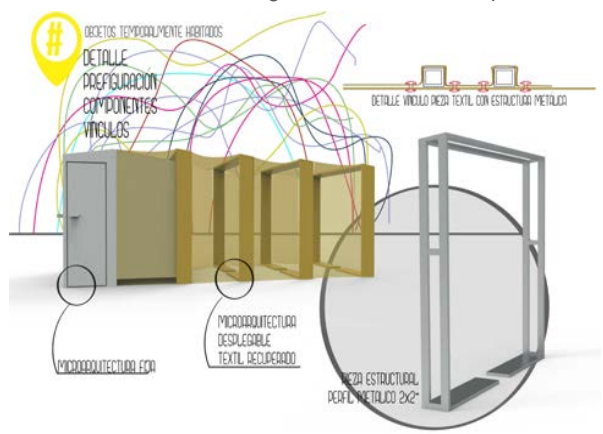

Imagen 12: propuesta física de espacio de servicio a insertar (imagen propia)

allá combinado dos de estas técnicas en un mismo tejido. Gracias a esto, se multiplican las posibilidades de recolectar energía. Según sus desarrolladores, la tela podría cargar los dispositivos a partir de algo tan simple como el viento que sopla en un día soleado.

Esta microarquitectura mutante está pensada para 2 situaciones del día, en donde el movimiento de gente es muy constante, cubrir situaciones de sol, lluvia y viento, tener acondicionamiento térmico en diferentes estaciones 
del año. (Imagen 9 y 10 y 11) Por la noche estas telas se recogen domóticamente, con algunos de los sistemas según Morales et al (2007) y se guardan a través de diferentes dispositivos y espacios quedando solo un prisma contenedor anti vandálico, lluminado e identificado como corresponde. Hay situaciones programáticas que van surgiendo en esta organización y la arquitectura propuesta se va acomodando para dar respuesta, conformándose un espacio espacio real que territorializa lo universitario, que es ágil, flexible. (imagen 12, 13, y 14)

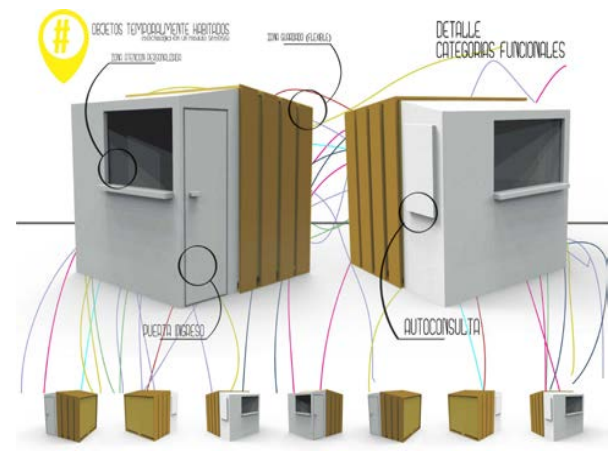

Imagen 13: propuesta física de espacio de servicio a insertar y sus cambios (imagen propia)

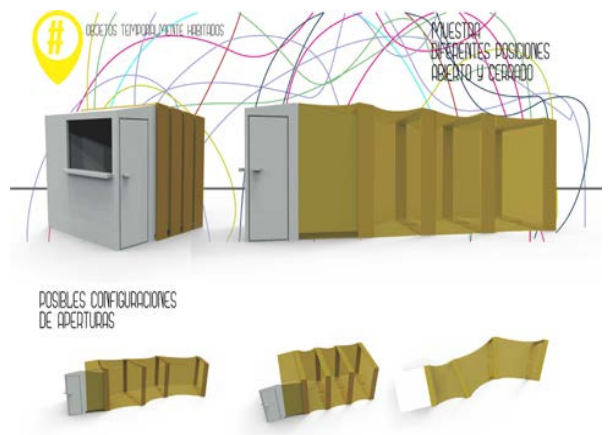

Imagen 14: propuesta física de espacio de servicio a insertar y sus pliegues y despliegues (imagen propia)

\section{RESULTADOS}

Consideramos que la difusión de las actividades de Cultura de la UNC y la articulación ciudad universitaria/ciudad capital es una herramienta de gran contribución al proceso de transformación de la Ciudad de Córdoba en una Smart City, por los evidentes aportes en materia de desarrollo sustentable e inclusión.
Se propuso el formato de microarquitectura con espacios habitables temporalmente, distribuidos por la ciudad, con un fuerte valor icónico, que simbolizan la llegada real, física y concreta de la Universidad a los barrios de la ciudad. La domótica, la arquitectura líquida con los materiales innovadores como herramienta de materialización y de mutación, hacen posible este proyecto y sus objetivos.

Los resultados abren una nueva pregunta ¿Se puede lograr que estas microarquitecturas de información y de servicio, no sean sólo el medio para incluir el uso de TIC, sino también para la mejora de la interacción de los usuarios de toda la ciudad? Esta deberá ser respondida en las futuras instancias de producción y puesta en funcionamiento del dispositivo.

\section{AGRADECIMIENTOS}

Agradecemos al laboratorio de Robótica de la UCEFyN, a los ingenieros electrónicos Lucio Madussi y Hugo DAllegre y a la Dra Arq. Sara Boccolini asesora en estructuras.

\section{REFERENCIAS}

Asociación Española de Domótica (CEDOM) (2007). Cuaderno de divulgación Domótica ( $2{ }^{a}$ ed.) Barcelona: Aenor.

Bauman, Zygmunt (2000). Modernidad Líquida. Mirta Rosenberg (trad). Argentina: Fondo de Cultura Económica. 2010. P.232. ISBN: 978-950-557-513-8

Bauman, Zygmunt (2007). Arte, ¿Líquido?.Francisco Ochoa de Michelena (trad). Madrid: Sequitur, 2007. P. 113. ISBN-13: 978-84-95363-36-7

Braceli, Miguel. Rescatado en septiembre de 20202 https://www.plataformaarquitectura.cl/cl/803173/zygmuntbauman-hacia-una-arquitectura-de-la-liquidez

Chiarella, M.; García Albarado, R. (2015). Folded compositions in architecture: spatial properties and materials, Nexus Network Journal, Springer.

De Los Santos Aransay, A., (2009). Diseño de interacción centrada en el usuario, Doctorado, Universidad de Vigo, España.

Gutiérrez-Rubí, A, (2010). Tecnopolítica. El uso y la concepción de las nuevas herramientas tecnológicas para la comunicación, la organización y la acción política colectivas. España.

MORALES, C.R.; et al. (2007). Domótica e inmótica. Madrid, Ed. Rama, $391 \mathrm{p}$.

Morales Guzmán, C. (2014). Diseño de sistemas transformables en las cubiertas tensadas. Madrid: Editoral académica Española.

Requena, G. (2019). Habitar híbrido: subjetividades e arquitetura do lar na era digital. Rio de Janeiro: Senac.

Novak, M. (2004). "TransTerraForm: Liquid Architectures and the Loss of Inscription," at CENTRIFUGE website. http://www.mat.ucsb.edu/ marcos/Centrifuge_Site/MainFram eSet.html, Recuperado el 10/28/2004.

Silva, C. A. (2005). Liquid architectures: Marcos Novak's territory of information. LSU Master's Theses. Recuperado de https://digitalcommons.Isu.edu/gradschool_theses/902

Romanutti, V. (Coord.) (2020). Plan de metas 2020/23 de la ciudad de Córdoba. Red Ciudadana. Recuperado de http://nuestracordoba.org.ar/sites/default/files/Las\%20metas $\% 20$ para \%20la\%20ciudad $\% 202020-2023$.pdf 